\title{
LA INTRODUCCIÓN DE LAS TEORÍAS DE E. ACHARIUS (1757-1819) EN LA LIQUENOLOGÍA ESPAÑOLA
}

\author{
Antonio GONZÁLEZ BUENO \& Víctor J. RICO
}

RESUMEN: El sistema taxonómico trazado por E. Acharius fue introducido en España por M. Lagasca y S.R. Clemente. La recepción del "Methodus..." (1803) se efectuó con anterioridad a Noviembre de 1806, a tenor de la correspondencia mantenida entre M. Lagasca y P. Lallave. La"Sinopsis..." (1814) fue utilizada con anterioridad a Junio de 1817, este texto fue distribuido por el algólogo sueco C.A. Agardh entre sus corresponsales A. Cabrera y F. Haenseler.

E. Acharius recibió líquenes españoles, herborizados por M. Lagasca, L. Dufour y P.K.A. Schousboë, y ejemplares americanos, de A.J. Cavanilles y J. Dombey, con anterioridad a 1807. Los materiales del "Ensayo..." (1807) de S.R. Clemente fueron recibidos por E. Acharius entre 1809 y 1814.

Se aportan los datos disponibles sobre la localización de los materiales citados por estos autores en sus trabajos liquenológicos.

Palabras clave: Liquenología, Acharius, España.

ABSTRACT: The system of lichen taxonomy outlined by E. Acharius was introduced in Spain by M. Lagasca and S.R. Clemente. As we now, from contemporary correspondence between M. Lagasca and P. Lallave, the "Methodus..." was received before November 1806. The Acharius "Synopsis..." (1814) was utilized before June 1817; the text was distribuited by the swedish algologist C.A. Agardh among his correspondents A. Cabrera and F. Haenseler.

Before 1807, spanish lichens were sent to E. Acharius by M. Lagasca, L. Dufour and P.K.A. Schousboë, and also, american material of A.J. Cavanilles and J. Dombey. E. Acharius received material from S.R. Clemente's work ("Ensayo..."; 1807) between 1809 and 1814. revised.

Information on location of herbarium material, cited by these mentioned autors is

Key words: Lichenology, Acharius, Spain.

\section{LA RECEPCIÓN DE LAS TEORÍAS DE E. ACHARIUS EN ESPAÑA}

El primer tratamiento sistemático del género Lichen L. fue publicado por E. Acharius en su "Methodus..." antes de fines Abril de 1803 (1); P. Lallave, a la sazón trabajando con E. Bompland en París, comunica a M. Lagasca la existencia del texto en carta fechada el 5 de Noviembre de 1806:

"Erik Achar que yo no sabia de su correspondencia con V. acaba de dar un "methodus quae omnes detectos lichenes secundum organa ampomorpha ad genera. 
1803". Sin embargo de esta fecha no ha mucho que es conocido aqui y han venido mui pocos ejemplares, sin embargo qe. son dos tomitos a la rustica me han costado 8 duros: la mayor parte de mis lichenes estan clasificados según su metodo. Tal vez en España no hay un solo ejemplar. Supongo qe. el mismo autor le habia hablado a V. sobre ello."

La obra era apenas conocida en los círculos botánicos franceses, pese a ello estaba ya al alcance de los botánicos españoles. M. Lagasca conocía los trabajos liquenológicos del sueco y, en 1805, publica sus primeras recolecciones siguiendo la terminología de E. Acharius (3); S.R. Clemente ordena el apéndice de su "Ensayo sobre las variedades de la vid...", publicado en 1807 (4), siguiendo el "Methodus...", así aparecen también dispuestos los materiales para la "Lichenographia Baetica" (5), inédita, para la que prepara un interesante prólogo:

"A vista del trabajo original de Acharius podria pensar alguno q. en este ultimo respecto resta ya muy poco por hacer. Yo, que solo he dedicado á la anatomia de los lichenes el tiempo preciso para fijar las especies andaluzas he visto el paso que los esfuerzos herculeos de aquel botanico fundador apenas han bastado a bosquejarla. El mismo lo reconoce así. Basta en efecto el alcance de los ojos, auxiliados alguna vez con una lente ordinaria, para asegurarse de que hay en los apotecios y en los talos partes, de que ó no se ha hablado todavia ó cuyas funciones nos son desconocidas, que estan aun sin determinar bien en gran numero de especies las metamorfosis que sufren unos y otros en el curso de la vegetacion, y como los efectos del agua, el calor, la luz, la humedad y la base sobre la que descansan, que muchas de las especies publicadas - estan aun sin caracterizar ó sin lugar determinado en el sistema, que las secciones en que las distribuye Acharius no siempre son exactas, que aun no se han determinado con precision los limites de algunos generos ni los que separan á la familia entera de los hongos y de las algas verdaderas, ó bien si se quiere, los eslabones que las unen y confunden con ellas." (6)

La entrada del "Methodus..." en España debió ser inmediata a su publicación, probablemente mediante envío del propio autor, quien mantenía ya relaciones epistolares con botánicos españoles (7).

S.R. Clemente supo de los trabajos en líquenes de E. Acharius durante su estancia en Londres, entre 1802 y 1803, mientras viajaba en compañía de F. Badia preparando su expedición por el Norte de Africa; allí contactó, entre otros, con K. Köning, quien le regaló un "Lichenographiae Sueciae Prodromus..." (1798), el ejemplar incluye una dedicatoria: "Dmo. Sm. de R. Clemente/ in amicitia tesserum/ Carolus Köning./Londini d. 10. Nov. 1802." (8). En este mismo viaje, S.R. Clemente adquirió una de las exsiccata distribuidas por J. Dickson (9).

El contacto de P. Lallave, un sacerdote de origen mexicano, con la obra de E. Acharius, se produce durante su estancia en París, en 1806; P. Lallave, a quien se debe la primera crítica de conjunto a la obra del liquenólogo sueco publicada en nuestro país, estudió y herborizó junto a E. Bompland y A.P. De Candolle (10), ellos le presentaron ante la Sociedad Filomática, donde P. Lallave defendió dos memorias, ambas sobre temas liquenológicos; lamentablemente, éstas no han llegado hasta 
nosotros, pero sí un resumen de las mismas que el autor comunica en carta a M. Lagasca de 19 de Junio de 1806:

"Esta semana se leerá el extracto de mis memorias en la Sociedad Filomática, Mr. Bompland y De Candolle haran la relación. La primera sobre el caracter clásico de las Usneas, y sobre las causas del fenómeno en que hago conmutar este caracter, la $2^{\mathrm{a}}$ es sobre la creacion de un genero nuevo que yo llamo Lacrinaria (para expresar el caracter) y que debe colocarse entre las secciones Lobaria y Sticta d'Acharius, yo he formado la Sticta dissecta d'Acharius, en que por mas que he hecho no he podido encontrar una cyphella que es el caracter de las Stictas; y de la Lobaria scrobiculata del mismo autor, en que hay ya rudimentos de ciphella, y a estas dos agrego yo el Lichen pulmonarius que Ach. ha colocado entre las Lobarias pero que tiene mas diferencias que afinidades en esta seccion. Mr. Bompland las ha hallado buenas y yo las imprimiria si tuviese dinero. Yo las he trabajado a ver si asi puedo ganar una patente de socio de esta academia en donde se leeran como digo esta semana." (11)

El 14 de Julio de 1807, E. Acharius firma el prólogo de su "Lichenographia Universalis...", aunque no parece probable que se editara antes de 1810 (12); en ella los líquenes citados o descritos de la Península Ibérica, se corresponden con materiales enviados por L. Dufour, M. Lagasca y P.K.A. Schousboë (13); A.J. Cavanilles y J. Dombey le proporcionan ejemplares americanos, esto confirma la relación de la "Escuela Cavanilles" con el botánico sueco (14). No tenemos constancia de la entrada en España de la "Lichenographia Universalis...", su distribución coincide con la expansión napoleónica, momentos poco favorables para el intercambio de noticias y publicaciones científicas.

No ocurrió así con la "Synopsis...", publicada en Octubre de 1814 (15), ésta sí llega pronto a nosotros, a través del algólogo sueco C.A. Agardh, quien mantenía contactos con F. Haenseler; éste comunica la publicación de la obra en carta a M. Lagasca de 26 de Abril de 1815:

"Este verano espero de el [C.A. Agardh] un paquete con plantas de Laponia, y el Synopsis Lichenum de Achario." (16)

Y meses más adelante, en Septiembre de 1815:

"El Sr. Agardh me prometió un Synopsis lichenum de Achario con plantas de Laponia pero yo no he visto lo primero, y de lo ultimo casi nada de Laponia y creo será porque el buque salió con mucha prisa, y lo efectuará en otra ocasión." (17)

F. Haenseler dispuso del texto, y se lo envió a M. Lagasca, antes de Junio de 1817, para entonces ya disponía de él A. Cabrera, y lo utilizaba en sus determinaciones conjuntas con P. Lallave (18); A. Cabrera obtuvo la obra por mediación de C. Agardh, con quien mantuvo un interesante epistolario (19).

S.R. Clemente, que ya mantenía correspondencia con E. Acharius, solicitó noticias sobre las publicaciones posteriores al "Methodus...", utilizando como intermediario al Conde de Gandía; así, en un borrador de carta, fechado el 28 de Agosto de 1815, escribe:

" Je profite de l'invitation que m'a faite Mr. le Compte de la Gandie, pour vous demandez des nouvelles sur la Botanique du Nord, et notamment sur vos travaux 
lichenographiques, dont je n'ai pas oi aucune notice positive apres la publication de votre Methodus, vos dernier ouvrages, que je entendire etre dejá bien repandues dans l'Europe, n'ayant encore penetréen dans l'Espagne. Si Mr. l'Ambassadeur m'avait annoncé sor depart quelques jours plutot, j'aunoieren le plaisir de vous envojer un petit paquet des lichens espagnols que nous savons ramasses ces derniers annes. Aussitot que les restes de nos collections achappeles aux lalands de la revolution seront reunir á Madrid, je saissizar la premiere ocássion pour vous en remettre la plus complette collection gut it me sont possible. Apres mon premier envoi de Lichenes je vous fit deus ou troix autres dont le dernier sur acompagné le Ensayo que je vous rement avec Mr. l'Ambassadeur ..." (20)

Durante los años 1814 y 1815, A. Cabrera, S.R. Clemente y P. Lallave, intensifican sus estudios liquenológicos; son los momentos de esplendor científico de este grupo de trabajo ubicado en Cádiz. En carta a M. Lagasca, de 8 de Septiembre de 1815, comenta P. Lallave:

"Algo de esto le toca al Compadre Clemente, que entre el y el bendito Achario me estan dando unos ratos de perros. Es el caso que el buen magistral se le ha antojado liquenear tambien y me ha buelto a meter en danza pasandome la mano con el Sinopsis de Achario posterior a la Lichenographia y que yo no habia visto. Tiene una porcion de generos nuevos (pues como todos los hermanos tiene este ...) y algunos de ellos a mi veer contra toda justificacion, pero en fin he tenido la satisfaccion o desconsuelo de veer pasados por imprenta algunos de mis lichenes nuevos, pero aun me quedan otros como despues dire." (21)

A. Cabrera, por su parte, escribe a M. Lagasca el 19 de Septiembre de 1815: "... lo bueno del entonces [el próximo envío] será la coleccion de liquenes, porque este Dn Pablo [Lallave] me trae todo entregado a ellos." (22)

La recepción de esta obra se produce, pues, por el puerto de Cádiz, hacia el verano de 1815; en 1816, P. Lallave presenta, en la sesión celebrada el 26 de Abril por la Sociedad Médico-Quirúrgica de Cádiz (23) un estudio crítico de las propuestas sistemáticas formuladas en el "Methodus..." y la "Sinopsis..." de E. Acharius, que le llevan a mantener: "Yo creo que en mucha parte debe preferirse el Methodus de Acharius a su Sinopsis" (24), sin duda por ajustarse más el primero a sus ideas de segregación de géneros expuestas en las memorias presentadas ante la Sociedad Filomática de París, en 1806.

\section{SOBRE LA LOCALIZACIÓN DE HERBARIOS}

En un intento de testificar las determinaciones de los materiales citados por estos autores, con miras a la elaboración de un censo bibliográfico de los líquenes españoles aún en preparación, hemos intentado localizar los herbarios donde se conserva el material recolectado por estos botánicos.

La aportación de M. Lagasca plantea pocos problemas, los táxones descritos con su material fueron publicados en la obra de E. Acharius; el herbario H-ACH (Helsinky) conserva ejemplares para respaldar estas descripciones (25). 
Más problemas plantea la localización de los pliegos utilizados por S.R. Clemente para redactar su "Flora Baetica...", de donde procede lo publicado por él o, en su nombre, por M. Colmeiro (26). Aunque E. Acharius utilizará el "Ensayo..." para la elaboración de su "Synopsis...", sabemos que no pudo testificar todo el material (27) y, según se desprende de la información facilitada por O. Vitikainen (in litt., 1986), no queda testimonio de ellos en $\mathrm{H}-\mathrm{ACH}$. Una parte importante del herbario de S.R. Clemente se conserva en MA (Madrid), pero no es suficiente para respaldar todas sus descripciones; una pequeña porción de pliegos fueron intercambiados por él con $\mathrm{F}$. Haenseler (28), éstos pasaron, con el resto del herbario del farmacéutico afincado en Málaga, a su discípulo P. Prolongo, y de él a la Sociedad Malagueña de Ciencias (29), ninguno de los pliegos conservados en esta colección corresponden a material tipo; otra porción, aún menor, está incluida entre los restos del herbario Boutelou conservados en Sevilla (30), éstos no se corresponden con plantas descritas en la "Flora Baetica...". La clave para la localización de este material parece estar en la colección Cabrera, el gaditano reconoce, a fines de 1809 (31), poseer los líquenes descritos por S.R. Clemente y aún alguno más, el herbario del Magistral Cabrera no ha sido localizado, según el testimonio de J. Pérez Lara (32), al menos parte de sus ejemplares estuvieron en poder de J.B. Chape, el único de sus discípulos botánicos reconocidos como tal por A. Cabrera, de su herbario sólo sabemos que, en 1891, se encontraba en el Instituto de Segunda Enseñanza de Cádiz, hoy no está allí.

Problemas similares plantea la localización del material utilizado por P.Lallave; buena parte de él debió quedar en la colección Cabrera, con quien trabajó durante su estancia en Cádiz, precisamente cuando leyó y publicó su "Descripción de algunos líquenes nuevos..." basándose en material mexicano, herborizado antes de su venida a España; gran parte de las colecciones mexicanas de P. Lallave estuvieron en poder de A.L. Lambert y, a su muerte, fueron vendidas en subasta (33), este material se encuentra hoy incorporado al Herbario del Conservatorio y Jardín Botánico de Ginebra; P. Geissler (in litt., 1986) nos comunica la ausencia de material tipo de los líquenes descritos por P. Lallave entre las colecciones conservadas en Ginebra. El problema queda pendiente de solución.

\section{AGRADECIMIENTOS}

Queremos hacer constar nuestra gratitud a P. Geissler (Ginebra), F. Muñoz Garmendia (Madrid), R. Moberg (Uppsala), F. Pelayo (Madrid) y O. Vitikainen (Helsinky) por la valiosa información proporcionada.

\section{NOTAS}

1. Entre Enero y Abril de 1803 las "sectio prior." (pp. I, III, I-LV, 1-152) y "sectio post." (pp. 153-393); J. Banks dispuso de una copia de la "sectio prior." el 28 de Abril de 1803. A éstas sigue un "Supplement" (pp. I-IV, 5-52) más tardío, entre Mayo y Diciembre de 1803. (Cf. F.A. Stafleu \& R.S. Cowan. Regnum Vegetabile, 94: 5. 1976). 
2. P. Lallave a M. Lagasca. Paris, 5-XI-1806 (Archivo del Real Jardín Botánico -Arch. RJB.leg. I, 56, 8, 8).

3. M. Lagasca. "Caracteres diferenciales de once especies nuevas de plantas y de otras dos poco conocidas." Variedades Ci. Lit. Artes, 4 (22): 212-217. 1805. En la pág. 214 comenta, tras proponer la sinonimia entre Parmelia roccella Ach. y Parmelia fucciformis Ach.: "Formo una especie de las plantas que Linneo, Acharius y demas botanicos han descrito en sus obras como dos especies distintas, sin duda porque no tuvieron la oportunidad, que yo logré de ver y observar en su país natal la variacion en la figura, color, \& c. de las partes en que se divide la fronde (thallus) de un mismo individuo..."

4. La primera edición en Madrid, por Villalpando, 1807; la segunda, del mismo año, por el Semanario de Agricultura, carece de las listas de plantas. Una traducción al francés, París, 1814; otra al alemán, Graz, 1821. Una tercera edición, en castellano, Madrid, 1879.

5. El manuscrito en Arch. RJB. leg. I, 53, 3, 3.

6. Op. cit. nota 5 .

7. Un borrador de carta entre M. Lagasca y E. Acharius se conserva en la Biblioteca de la Universidad de Uppsala (R. Moberg, in litt., 1986).

8. El ejemplar se conserva en la Biblioteca en la Facultad de Farmacia de la Universidad Complutense de Madrid.

9. S.R. Clemente comunica así la compra del exciccatum a M. Lagasca: "Me he resuelto á comprar á Dickson una coleccioncita de los líquenes que nos faltan que son muchos (...) Esto me parece todavia mas necesario que los libros." (S.R. Clemente a M. Lagasca. Londres, 3I-1803. -Arch. RJB. I, 56, 4, 18-).

10. Alusiones como "Mañana que voi a liquenear a casa de Bompland" no son raras en la correspondencia cursada entre P. Lallave y M. Lagasca durante la estancia del primero en Paris. (Cf., e.g., P. Lallave a M. Lagasca. Paris, 5-XI-1806. -Arch. RJB. leg. I, 56, 8, 8-.

11. P. Lallave a M. Lagasca. Paris, 19-VI-1806. (Arch. RJB. leg. I, 56, 8, 39).

12. Al menos con tal título, aunque sí las plantas en ella descritas, alguna de las cuales están válidamente publicadas por Acharius in Luyken (1809) (cf. F.A. Stafleu \& R.S. Cowan. Regnum Vegetabile, 94: 5. 1976).

13. Y quizás también H.F. Link; la referencia "Lusatia. Link" de la pág. 501, para Borrera villosa, debe entenderse "Lusitania. Link". (Cf. O. Vitikainen. "Erik Acharius." In: Lichenographia Universalis. A reedition. 1976).

14. Muy significativo el comentario que acompaña a Usnea melaxantha (Ach.) Ach. en la Lichenographia Universalis... pág. 618: "sed Specimina a me visa et b.m. Amico Cavanilles missa, ad Port Egmont lecta...". El texto publicado en 1810, pero probablemente escrito antes 
de 1807. Sobre la "Escuela de Cavanilles" cf. A. González Bueno. "Los estudios criptogámicos en España (1800-1820): una aproximación a la Escuela Botánica de A.J. Cavanilles." Llull, 11: 51-74. 1988.

15. F. Haenseler a M. Lagasca. Málaga, 26-IV-1815 (Arch. RJB. leg. I, 56, 7, 5).

16. F. Haenseler a M. Lagasca. Málaga, 6-IX-1815 (Arch. RJB. leg. I, 56, 7, 7). En el mismo sentido Ib. Málaga, 4-VI-1816 (Arch. RJB. leg. I, 56, 7, 8).

17. F. Haenseler a M. Lagasca. Málaga, 18-VI-1817 (Arch. RJB. leg. I, 56, 7, 9).

18. P. Lallave a M. Lagasca. Cádiz, 8-VII-[1815] (Arch. RJB. leg. I, 56, 8, 5). La carta, aunque sin año, debe pertenecer a 1815, en 1814 no podría aludir al "Sinopsis..." de E. Acharius, y en 1816 ya había leído su memoria ante la Sociedad Médico-Quirúrgica de Cádiz (26-IV-1816), aquí en preparación.

19. Correspondencia conservada en la Universidad de Lund; de ella y de otros manuscritos de A. Cabrera, uno de nosotros (A. González Bueno) prepara una edición crítica.

20. S.R. Clemente a E. Acharius. [Madrid], 28-VIII-1815 (Arch. RJB. leg. I, 58, 3, 15).

21. P. Lallave a M. Lagasca. Cádiz, 8-XI-1815 (Arch. RJB. leg. I, 56, 8, 5).

22. A. Cabrera a M. Lagasca. Cádiz, 19-IX-1815 (Arch. RJB. leg. I, 56, 3, 31).

23. P. Lallave. "Descripción de algunos líquenes nuevos." Periódico Soc.Med.-Quirur.Cádiz, 1: 99-117. 1820.

24. Op. cit. nota 23, pág. 108.

25. Cf. H.T. Lumbsch. "The identity of Diploschistes gypsaceus." Lichenologist, 20 (1): 1924. 1988.

26. M. Colmeiro. "Enumeración de las criptógamas de España y Portugal." Rev.Progr.Ci., 1718: 1-260. 1867.

27. Careció de ejmplares de Lecanora turneri Clem., "proteren dubius an Lecideis vel Gyrophoris potius as sociari debeat, specimine ejusdem a me non viso" (E. Acharius. Synopsis... Lund, 1814, pág. 68) y de Parmelia caricae Clem., "Ad Lecanora nec Parmelia hanc a me nodum visam speciem, quam ut crustaceum describit cel. Clemente" (E. Acharius. Op. cit. pág. 100) entre otros.

28. "A nuestro Clemente muchas cosas y mucho desearia me determinasen Vds, las plantas restantes, así como los liquenes y piedras que les remiti esta pasado..." (F. Haenseler a M. Lagasca. Málaga, 6-I-1815 -Arch. RJB. leg. I, 56, 7, 8-) y algo molesto, algunos meses después: "Veremos si en mi pequeña expedicion encuentro algunos liquenes dignos de la atencion de nuestro amigo Clemente..." (Ib. Málaga, 24-V-1815-Arch. RJB. leg. I, 56, 7, 9). 
29. Cf. M. Laza Palacios. "Notas sobre un herbario de plantas andaluzas de D. Simón de Rojas Clemente y Rubio." Bol. Soc. Esp. Hist. Nat., 40: 263-298. 1942.

30. Cf. S. Silvestre \& J. García-Rowe. "Líquenes en los Herbarios Boutelou, de la Universidad de Sevilla y del Antiguo Museo de Historia Natural de Sevilla". Collect. Bot. (Barcelona), 13 (1): 375-380. 1982.

31. "Entre otras tengo como otros 20 cistos (...) con todos los Líquenes de la Lista de Clemente, y alguno otro mas." (A. Cabrera a M. Lagasca. Sevilla, 23-IX-1809 -Arch. RJB. leg. I, 56, 3, 22).

32. "... el Sr. D. Juan B. Chape, quien con gran bondad me (...) facilitó el estudio (...) de su herbario, ya bastante deteriorado, en el que se encuentran algunos ejemplares que fueron recogidos por el Magistral Cabrera." (J.M. Pérez-Lara. "Flórula Gaditana. Pars prima." Anal. Soc. Esp. Hist. Nat., 15: 348-475. 1866. El texto citado en pág. 15).

33. Cf. H.S. Miller. "The Herbarium of Aylmer Bourke Lambert. Notes on its adquisition, dispersal, and present whereabouts." Taxon, 19: 489-656. 1970. En pág. 529: "Lot. 177. Miscellaneous Mexican Plants by Lallave, Staples, and others, a small collection."

(Aceptado para su publicación en Junio de 1.990 )

Dirección de los autores: A. GONZÁLEZ BUENO: Dpto. Farmacia y tecnología Farmacéutica. V.J. RICO: Dpto. Biología Vegetal II. Facultad de Farmacia. Universidad Complutense. 28040 Madrid. 